\title{
Barriers to Women Entering Surgical Careers: A Global Study into Medical Student Perceptions
}

\author{
I. H. Marks ${ }^{1}$ (1) - A. Diaz ${ }^{2}$ - M. Keem ${ }^{3} \cdot$ Seyedeh-Sanam Ladi-Seyedian $^{4}$ - G. S. Philipo ${ }^{5}$ \\ H. Munir ${ }^{6}$ - T. I. Pomerani ${ }^{7}$ H. M. Sughayer ${ }^{8}$ - N. Peter ${ }^{9}$ C. Lavy ${ }^{9}$ D. C. Chang ${ }^{10}$
}

Published online: 15 October 2019

(C) The Author(s) 2019

\begin{abstract}
Background Barriers to female surgeons entering the field are well documented in Australia, the USA and the UK, but how generalizable these problems are to other regions remains unknown.

Methods A cross-sectional survey was developed by the International Federation of Medical Students' Associations (IFMSA)'s Global Surgery Working Group assessing medical students' desire to pursue a surgical career at different stages of their medical degree. The questionnaire also included questions on students' perceptions of their education, resources and professional life. The survey was distributed via IFMSA mailing lists, conferences and social media. Univariate analysis was performed, and statistically significant exposures were added to a multivariate model. This model was then tested in male and female medical students, before a further subset analysis by country World Bank income strata.

Results 639 medical students from 75 countries completed the survey. Mentorship [OR 3.42 (CI 2.29-5.12) $p=0.00$ ], the acute element of the surgical specialties [OR $2.22(\mathrm{CI} 1.49-3.29) p=0.00]$, academic competitiveness [OR 1.61 (CI 1.07-2.42) $p=0.02$ ] and being from a high or upper-middle-income country (HIC and UMIC) [OR 1.56 (CI $1.021-2.369) p=0.04]$ all increased likelihood to be considering a surgical career, whereas perceived access to postgraduate training [OR $0.63(\mathrm{CI} 0.417-0.943) p=0.03$ ], increased year of study [OR 0.68 (CI $0.57-0.81) p=0.00$ ] and perceived heavy workload [OR 0.47 (CI 0.31-0.73) $p=0.00$ ] all decreased likelihood to consider a surgical career. Perceived quality of surgical teaching and quality of surgical services in country overall did not affect students' decision to pursue surgery. On subset analysis, perceived poor access to postgraduate training made women $60 \%$ less likely to consider a surgical career [OR $0.381(\mathrm{CI} 0.217-0.671) p=0.00$ ], whilst not showing an effect in the men [OR 1.13 (CI 0.61-2.12) $p=0.70$. Concerns about high cost of training halve the likelihood of students from low and low-middleincome countries (LICs and LMICs) considering a surgical career [OR 0.45 (CI 0.25-0.82) $p=0.00$ ] whilst not demonstrating a significant relationship in HIC or UMIC countries. Women from LICs and LMICs were $40 \%$ less likely to consider surgical careers than men, when controlling for other factors [OR $0.59 \mathrm{CI}(0.342-1.01 p=0.053$ ].

Conclusion Perceived poor access to postgraduate training and heavy workload dissuade students worldwide from considering surgical careers. Postgraduate training in particular appears to be most significant for women and cost of training an additional factor in both women and men from LMICs and LICs. Mentorship remains an important and modifiable factor in influencing student's decision to pursue surgery. Quality of surgical education showed no effect on student decision-making.
\end{abstract}

Electronic supplementary material The online version of this article (https://doi.org/10.1007/s00268-019-05199-1) contains supplementary material, which is available to authorized users.

I. H. Marks

isobel.h.marks@gmail.com
Department of Primary Care and Public Health, Imperial College, London, UK 


\section{Introduction}

Surgery has long been considered an old boys' club [1, 2]. In the UK in 2013, women made up less than $10 \%$ of consultant surgeons, while a majority of new medical graduates responding to a survey on career intentions perceived a surgical career to be unwelcoming to women [3]. Proportions of women and graduate students entering medical school are increasing, but they are less likely to pursue a career in surgery, prompting appeals in the literature for renewed emphasis on promoting surgery to medical students [2-4].

Much of the literature assessing motivations to pursue surgical careers is restricted to a high-income country context, wherein a lack of female role models and a hostile culture towards women are frequently cited as key barriers to pursuing a surgical career as a woman [2, 4-7]. Yet while significant work has been completed in Western countries on the benefits of mentorship and positive role models $[2,4,8,9]$, there is little evidence that this trend is generalizable worldwide; indeed, work that has examined global generalizability of such barriers has found significant regional variation [8]. Furthermore, some studies from high-income countries dispute that a lack of role models or lifestyle factors influence surgical career choices in women relative to men, instead underscoring a hostile surgical culture and a perceived "surgical personality" as deterrent factors specific to women [10]. Workload and "work-life balance" have also frequently been cited as deterrents for women undertaking a surgical career $[11,12]$. There is a paucity of research examining factors influencing a decision to pursue a surgical career in low- and middle-income countries.

With one-third of surgical disease burden occurring in low- and middle-income countries, there is a strong

2 Virginia Commonwealth University School of Medicine, Richmond, VA, USA

3 Royal Melbourne Hospital, Melbourne, Australia

4 Students' Scientific Research Center (SSRC), Tehran University of Medical Sciences, Tehran, Iran

5 School of International Education, Southern Medical University, Guangzhou, China

6 Jinnah Hospital, Lahore, Pakistan

7 Medicine and Surgery, School of Human Health Sciences, University of Florence, Florence, Italy

8 Faculty of Medicine, Al-Quds University, East Jerusalem, Palestine

$9 \quad$ Nuffield Department of Orthopaedics Rheumatology and Musculoskeletal Sciences, University of Oxford, Oxford, UK

10 Department of Surgery, Massachusetts General Hospital, Boston, USA impetus to study barriers to entry for surgical careers given increased interest in increasing surgical workforce in these countries by the year 2030 [13, 14]. Therefore, we composed a survey on attitudes and barriers to pursuing a surgical career and undertook a cross-sectional analysis with the resultant data, aiming to examine discrepancies between countries of differing income strata and between genders (online Supplemental Material).

\section{Methods}

In collaboration with the University of Oxford, we developed a cross-sectional, mixed methods survey. The survey was created by a focus group of medical students from Asia, Africa, Europe and the Americas. Over a series of online meetings, we determined focus areas and performed face validation of the survey. A literature review was performed to attest for criterion validity, and all previously surveyed areas of interest studied regarding medical students interested in surgery were included in the final survey. The survey contained 37 questions arranged in five sections: basic demographics, including medical school location and length of study, as well as questions relating to the following thematic areas: education, resources, perceptions and profession-related. Equivalent-form reliability was considered and tested. The language of the survey was deemed suitable for non-native speakers by trialling on students from four different continents. Equivalent-form reliability was tested using different question wordings relating to access to postgraduate training and surgical teaching. The Cronbach's alpha test was used to determine internal validity of study questions. The questions were measured on a Likert scale ( 1 strongly disagree, 2 disagree, 3 neither agree of disagree, 4 agree, 5 strongly agree), which was converted to binary on multivariate analysis. Analysis was completed using Intercooled Stata software, version 13.0 (StateCorp College Station, TX, USA).

The survey was hosted on a web-based survey platform via the University of Oxford and was distributed via multiple mailing and social media of the International Federation Medical Student Association (IFMSA) and its Global Surgery Working Group. The study was open to all medical students and was entirely anonymous and confidential. Questionnaires from graduates after their internship year (defined as one year post medical school) were excluded, in keeping the inclusion criteria the same as IFMSA membership. The outcome of interest was consideration of pursuing a surgical career (yes/no). A univariate analysis was performed of individual questions against this outcome. A logistic regression model was then constructed with all factors that were statistically significant on univariate analysis, plus other questions of interest that were 
added adjustment, including workforce density in country. A stepwise progression was performed, and factors that showed no association were withdrawn from the model. The final model contained mentorship, acute care element of surgical careers, cost of postgraduate training, workload, academic competitiveness, academic competitiveness, year of study and World Bank Income status, which was divided into HICs and UMICs ad LICs and LMICs. Interactions were tested in the model with gender. Subsequently, a subgroup analysis was performed on male and female participants separately and then by income strata.

\section{Results}

Our study population consisted of 639 medical students from 75 countries (Fig. 1). The demographic profile of respondents is given in Table 1. Overall, 58.5\% $(n=374)$ of respondents were female. $4.5 \%(n=29)$ of respondents were from low-income countries (LICs), 27\% $(n=172)$ from lower-middle-income countries (LMICs), $20 \%$ from upper-middle-income countries (UMICs) and $48 \%$ $(n=308)$ from high-income countries (HICs). Table 1 shows student's intentions to pursue a surgical career by the stage of training, separating male and female respondents. The univariate analysis of questions is displayed in Table 2, and multivariable logistic regression results are shown in Table 3.

On multivariate analysis, mentorship [OR 3.42 (CI 2.29-5.12) $p=0.00]$, the acute element of the surgical specialties [OR 2.22 (CI 1.49-3.29) $p=0.00$ ], academic competitiveness [OR 1.61 (CI 1.07-2.42) $p=0.02$ ] and being from a high or upper-middle-income country (HIC and UMIC) [OR 1.56 (CI 1.021-2.369) $p=0.04$ ] all increased likelihood to be considering a surgical career. Perceived access to postgraduate training [OR 0.63 (CI $0.417-0.943) p=0.03$ ], increased year of study [OR 0.68 (CI 0.57-0.81) $p=0.00]$ and perceived heavy workload [OR 0.47 (CI 0.31-0.73) $p=0.00]$ all decreased likelihood to consider a surgical career. Perceived educational quality however showed no affect on students' decision to consider a surgical career when tested using several variations of education-related questions. It was thus excluded from the model. The gender of the mentor was significantly associated with pursuing a surgical career in the univariate analysis but became insignificant when controlling for mentorship as a whole on multivariate analysis, and this question was also thus excluded from the final model.

On subset analysis, perceived poor access to postgraduate training made women $60 \%$ less likely to consider a surgical career [OR 0.381 (CI 0.217-0.671) $p=0.00$ ], whilst not showing an effect on men [OR 1.13 (CI 0.61-2.12) $p=0.70$. Concerns about high cost of training halved the likelihood of students from low and low-middleincome countries (LICs and LMICs) considering a surgical career [OR 0.45 (CI 0.25-0.82) $p=0.00$ ] whilst not demonstrating a significant relationship in HIC or UMIC countries. Women from LICs and LMICs were $40 \%$ less likely to consider surgical careers than men, when controlling for other factors [OR 0.59 CI (0.342-1.01 $p=0.053]$. Figure 2 demonstrates the raw data answers from the questions "How do you rate access to postgraduate surgical training programmes?" dividing answers by income strata, whilst Fig. 3 shows the responses to this question by both gender and income strata as a percentage of the total responders within each category. Finally, Fig. 4 demonstrates the regression coefficients for the association of access to postgraduate training opportunities versus decision to pursue surgery as a career, with coefficients greater than one representing a positive association, whilst controlling for the other questions described in the multivariate analysis.

Comparing groups by World Bank income strata in country of study, concerns about high cost of training halved the likelihood of students from low and low-middleincome countries (LICs and LMICs) considering a surgical career [OR 0.45 (CI 0.25-0.82) $p=0.00$ ] whilst not demonstrating a significant relationship in students from HIC or UMIC countries. Women from LICs and LMICs were $40 \%$ less likely to consider surgical careers than men, when controlling for other factors [OR $0.59 \mathrm{CI}$ (0.342-1.01 $p=0.053]$.

A number of questions were used to test internal survey internal validity, using the Cronbach's alpha test. "Surgery is taught well at my university" and "How would you rate the quality of your surgical teaching overall?" demonstrated a reliability coefficient of 0.7741 (good). "Do you think that doctors in your country have good access to postgraduate surgical" and "How do you rate access to postgraduate surgical training programmes?" were tested, resulting in a reliability coefficient 0.5464 (acceptable).

\section{Discussion}

This study demonstrates that a wide variety of factors influence student's decisions pursue a surgical career. Mentorship, the acute element of surgical care and academic competitiveness increased students' likelihood to consider surgical career across gender and income strata, whereas concerns over access to postgraduate training, heavy workload and progression through medical school all decreased likelihood to consider surgery. Access to postgraduate training in particular demonstrated a gender interaction and reduced women's likelihood to consider surgical careers by around $60 \%$ when controlling for other 


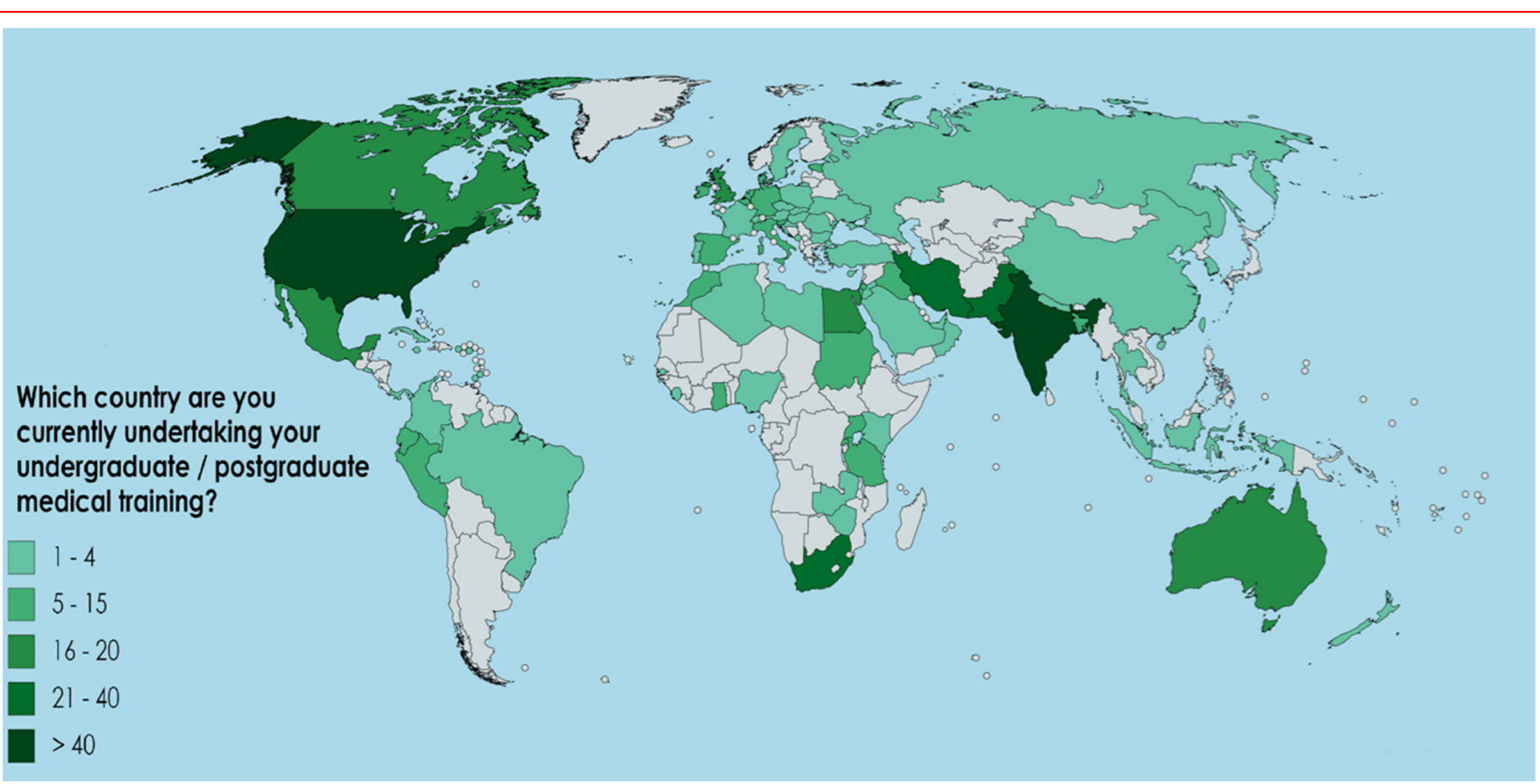

Fig. 1 Country distribution of student responses

Table 1 Population characteristics

\begin{tabular}{llll}
\hline Variable & $\mathrm{N}(\%)$ & Female & Male \\
\hline Sample size & 639 & $374(58.5 \%)$ & $265(41.5 \%)$ \\
Proportion considering a career in surgery & $67.70 \%$ & $243(65 \%)$ & $188(71 \%)$ \\
Average per cent of medical school completed & $71.60 \%$ & $72.7 \%$ & $70.1 \%$ \\
World bank income level & Percentage of total population & Female & Male \\
\hline Low & $29(4.5 \%)$ & $7(24.14 \%)$ & $22(75.86 \%)$ \\
Lower-middle & $172(27.0 \%)$ & $106(61.63 \%)$ & $66(38.37 \%)$ \\
Upper-middle & $130(20.3 \%)$ & $65(50.00 \%)$ & $65(50.00 \%)$ \\
High & $308(48.2 \%)$ & $196(63.64 \%)$ & $112(36.36 \%)$ \\
\hline
\end{tabular}

factors $(p=0.0281)$. Whilst the importance of mentorship is in concordance with existing literature, perceived poor access to postgraduate training preventing women from considering surgical careers is a new finding. This finding was generalizable across income groups and may demonstrate more subtle biases throughout the surgical application process.

Whilst many global surgery programmes focus on teaching surgical skills, our study has demonstrated that perceived quality of surgical teaching does not affect student's decisions to pursue surgery, nor do concerns regarding the overall surgical quality in their country of study. It may be that focusing global surgical programmes on improving access to postgraduate opportunities or lowering the cost of postgraduate training may be more effective in bringing medical students into surgical careers in these settings. A concerted effort into improving mentorship structures would likely also be beneficial, given that in our study, agreement with the importance of mentorship made students four times more likely to consider a surgical career. The effect of year group and progression through medical school is in accordance with the literature. Surgery is widely regarded as a popular specialty of choice amongst first year medical students [15], but importantly most students change their specialty choices, regardless of initial interest [16]. 
Table 2 Univariate analysis

\begin{tabular}{ll}
\hline Consider surgery & $P$ value \\
\hline Gender & 0.113 \\
Mentorship & $\mathbf{0 . 0 0 0}$ \\
Acute element & $\mathbf{0 . 0 0 0}$ \\
Postgraduate training & $\mathbf{0 . 0 2 5}$ \\
Training cost & $\mathbf{0 . 0 7 2}$ \\
Public perception of surgeons & 0.086 \\
Academic competitiveness & $\mathbf{0 . 0 0 8}$ \\
Access to resources & 0.516 \\
Year of study & $\mathbf{0 . 0 0 0}$ \\
Practical surgical experience & $\mathbf{0 . 0 0 1}$ \\
Workload of surgeons & $\mathbf{0 . 0 0 6}$ \\
World bank income & 0.119 \\
Quality of surgical teaching & 0.277 \\
Morale amongst surgeons & 0.246 \\
Quality of surgery in my country & 0.736 \\
Political issues or security threats & 0.562
\end{tabular}

Bold values indicate stastically significant result $(p<0.05)$

There has been a growing interest in looking at women in surgery. With almost all literature examining European and American trainees, our study is the first attempt to see whether such findings are consistent worldwide and across income stratas. Access to and cost of postgraduate training made women in specific regions and income groups significantly less likely to pursue a surgical career, and on subset analysis of the LIC and LMIC students, being female made students $40 \%$ less likely to consider surgery, independent of other factors (see Tables 4, 5) Trials of women-only scholarships for postgraduate surgical training may be useful in eliminating financial barriers from young female doctors, and further work should be pursued to elicit the causes of this perceived financial impediment.

The finding that perceived educational quality bears no significance on students' decision to pursue a surgical

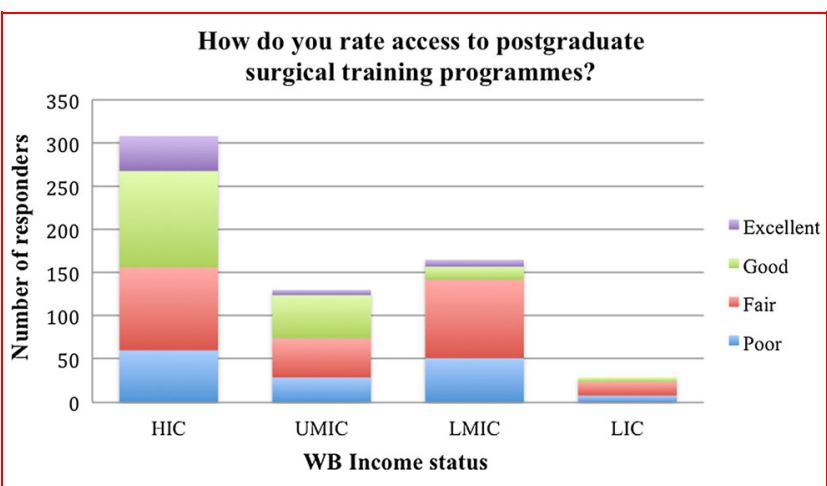

Fig. 2 Answers to "How do you rate access to postgraduate surgical training programmes?" by income strata of country of study

career has important implications for the success of mission trips from HICs focusing on teaching surgical skills. It suggests that such efforts may be better focused on increasing access to sustainable postgraduate training programs, or else offering meaningful mentorship opportunities. However, whilst we have demonstrated that gender has an effect on decisions to pursue surgery, there may also be interactions with other factors, such as the income status of the students' country of study. Women therefore should not be treated as a global, homogenous group.

It is important that medical educators consider how medical school-based experiences, interactions with mentors and perceived postgraduate opportunities, impact students' specialty choices and decision-making. Mentorship clearly plays an important role in the decisions of both men and women, but access to and funding for postgraduate training may also play an important role in encouraging women to take up this career path.

This study was limited by its selection bias. IFMSA is made up of motivated and internationally active students and may not be generalizable to the true medical student population. However, the authors believe that this bias is limited by the fact that this was true in both male and

Table 3 Multivariate analysis

\begin{tabular}{llll}
\hline Consider surgery-total population & Odds ratio & $P$ value & Confidence interval \\
\hline Mentorship & 3.423 & $\mathbf{0 . 0 0 0}$ & $(2.291-5.117)$ \\
Training cost & 0.745 & 0.173 & $(0.488-1.138)$ \\
Acute element & 2.216 & $\mathbf{0 . 0 0 0}$ & $(1.492-3.291)$ \\
Access to postgraduate training & 0.627 & $\mathbf{0 . 0 2 5}$ & $(0.417-0.943)$ \\
Academic competitiveness & 1.610 & $\mathbf{0 . 0 2 1}$ & $(1.073-2.417)$ \\
Year of study & 0.676 & $\mathbf{0 . 0 0 0}$ & $(0.566-0.807)$ \\
Workload of surgeons & 0.476 & $\mathbf{0 . 0 0 1}$ & $(0.312-0.727)$ \\
WB income (HIC/UMIC or LMIC/LIC) & 1.555 & $\mathbf{0 . 0 4 0}$ & $(1.021-2.369)$ \\
\hline
\end{tabular}

Bold values indicate stastically significant result $(p<0.05)$ 


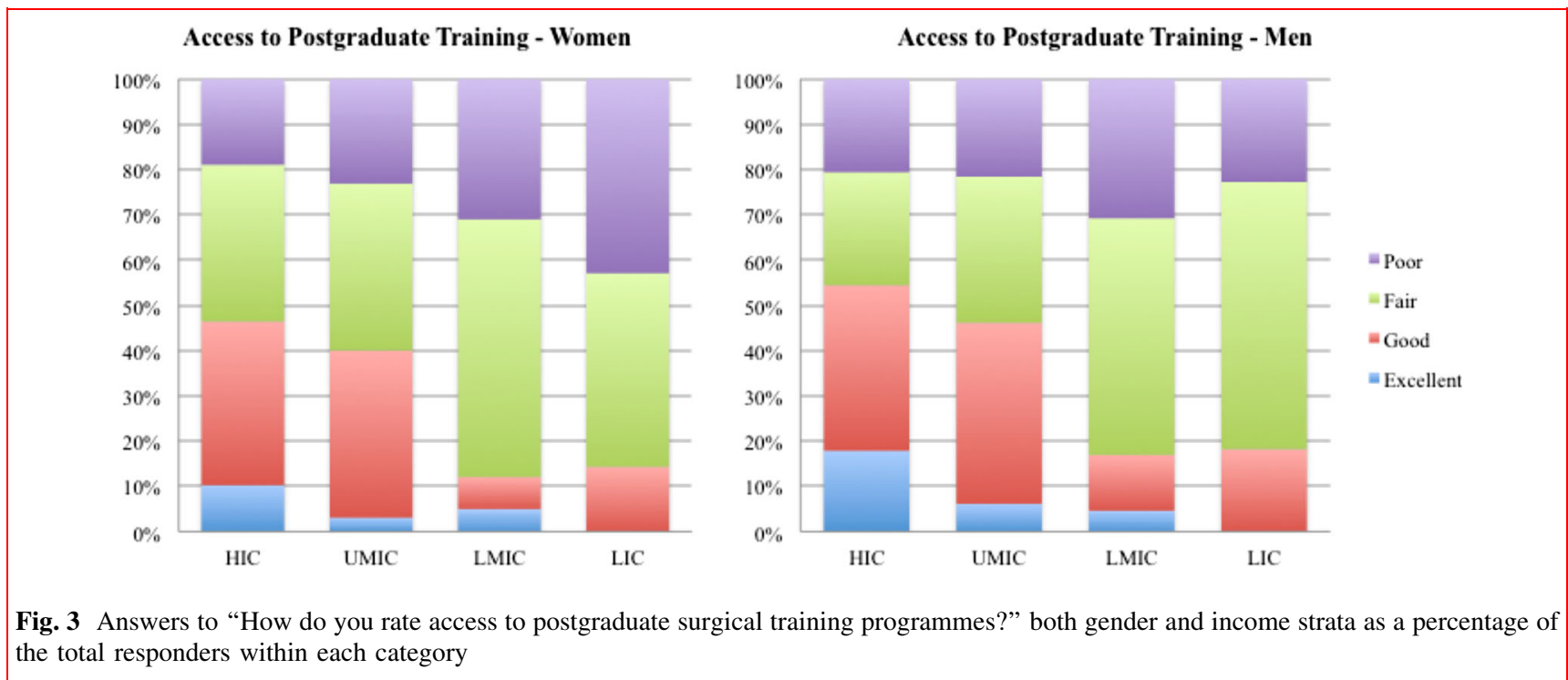

Fig. 3 Answers to "How do you rate access to postgraduate surgical training programmes?" both gender and income strata as a percentage of the total responders within each category

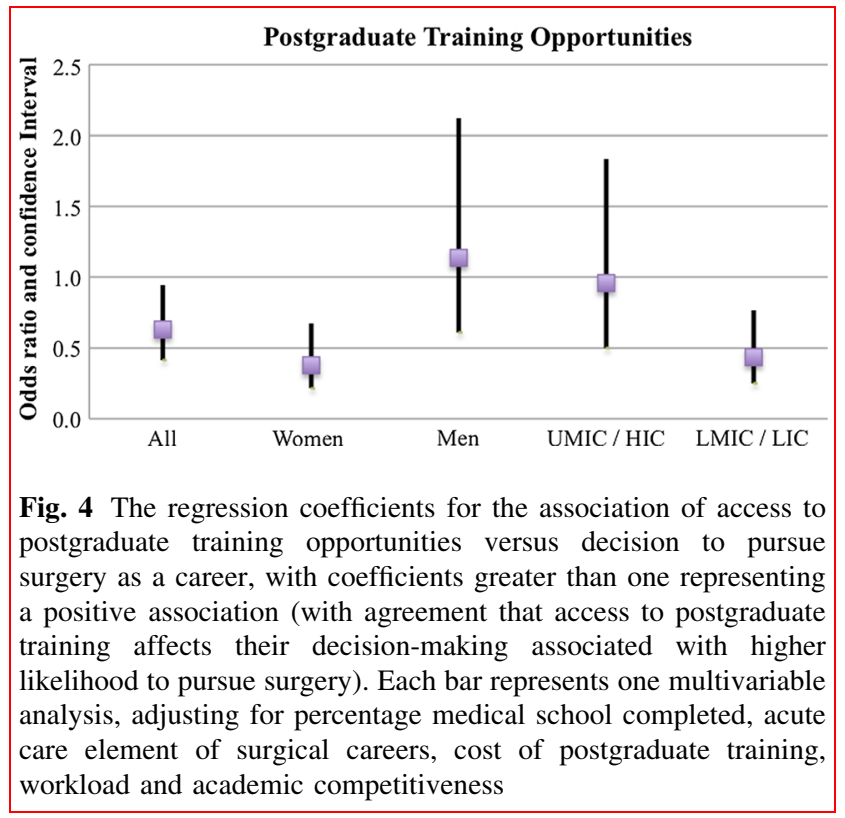

female populations, and also that no questions pertained to travel or global health, as would preference students attending international events. The study is also limited by disproportionate representation of certain countries. This has led to larger confidence intervals especially on subset analysis and may lead to type II error. However, by targeting existing medical students, this study eliminates any recall bias by of questioning surgical trainees. This study focused entirely on pursuing surgical careers, and does not question participants on their consideration of anaesthesia or obstetrics, both of which are important for reducing the burden of surgical disease. Further work looking into these areas would be beneficial.

\section{Conclusion}

This study is the first of its kind globally to quantify differences in barriers to surgical careers in medical students worldwide. Whilst we have been unable to draw firm conclusions on all of the driving forces behind decisionmaking, we have determined many patterns and areas for further study and potential investment. Mentorship the acute element of surgical care and academic competitiveness were positively impactful on medical students' decision to pursue a surgical career across genders and income strata, while perceived poor access to postgraduate training and heavy workload made women in particular less likely to consider a surgical career. While there are some similarities amongst women worldwide, it is important to ensure that cultural assumptions and evidence amassed in high-income country are not generalized to other regions without evidence of consistency in behaviour or perception. There is considerable interaction between income strata and gender differences, as well as different factors that may be targeted to encourage female trainees in different regions. Emphasis on effective mentorship, improving postgraduate opportunities and reducing workload may all contribute to improving opportunities for medical students, and particularly women to pursue surgical careers worldwide. 
Table 4 Multivariate by gender-(i) multivariate analysis in female students and (ii) multivariate analysis in male students

\begin{tabular}{|c|c|c|c|}
\hline Consider surgery-women & Odds ratio & $P$ value & Confidence Interval \\
\hline \multicolumn{4}{|l|}{ (i) } \\
\hline Mentorship & 3.914 & 0.000 & $(2.294-6.678)$ \\
\hline Training cost & 0.570 & 0.057 & $(0.320-1.017)$ \\
\hline Acute element & 2.939 & 0.000 & $(1.730-4.994)$ \\
\hline Access to postgraduate training & 0.381 & 0.001 & $(0.217-0.671)$ \\
\hline Academic competitiveness & 1.815 & 0.031 & $(1.057-3.118)$ \\
\hline Year of study & 0.788 & 0.053 & $(0.620-1.004)$ \\
\hline Workload of surgeons & 0.558 & 0.046 & $(0.314-0.990)$ \\
\hline WB income (HIC/UMIC or LMIC/LIC) & 2.084 & 0.014 & $(1.163-3.734)$ \\
\hline Consider surgery-men & Odds ratio & $P$ value & Confidence interval \\
\hline \multicolumn{4}{|l|}{ (ii) } \\
\hline Mentorship & 3.519 & 0.000 & $(1.834-6.754)$ \\
\hline Training cost & 0.899 & 0.752 & $(0.464-1.742)$ \\
\hline Acute element & 1.665 & 0.112 & $(0.888-3.123)$ \\
\hline Access to postgraduate training & 1.134 & 0.695 & $(0.606-2.122)$ \\
\hline Academic competitiveness & 1.266 & 0.477 & $(0.661-2.423)$ \\
\hline Year of study & 0.568 & 0.000 & $(0.428-0.752)$ \\
\hline Workload of surgeons & 0.402 & 0.007 & $(0.208-0.777)$ \\
\hline WB income (HIC/UMIC or LMIC/LIC) & 1.180 & 0.617 & $(0.617-2.255)$ \\
\hline
\end{tabular}

Bold values indicate stastically significant result $(p<0.05)$

Table 5 Multivariate by income strata-(i) multivariate analysis in students studying in HICs and UMICs and (ii) multivariate analysis in students studying in LMICs and LICs

\begin{tabular}{|c|c|c|c|}
\hline $\mathrm{UMIC} / \mathrm{HIC}$ & Odds ratio & $P$ value & Confidence interval \\
\hline \multicolumn{4}{|l|}{ (i) } \\
\hline Mentorship & 5.723 & 0.000 & $(3.107-10.543)$ \\
\hline Training cost & 1.136 & 0.704 & $(0.588-2.197)$ \\
\hline Acute element & 2.504 & 0.002 & $(1.409-4.448)$ \\
\hline Access to postgraduate training & 0.961 & 0.904 & $(0.502-1.837)$ \\
\hline Academic competitiveness & 1.654 & 0.093 & $(0.919-2.978)$ \\
\hline Year of study & 0.577 & 0.000 & $(0.433-0.769)$ \\
\hline Workload of surgeons & 0.460 & 0.019 & $(0.240-0.881)$ \\
\hline Gender & 0.910 & 0.754 & $(0.504-1.643)$ \\
\hline LMIC/LIC & Odds ratio & $P$ value & Confidence interval \\
\hline \multicolumn{4}{|l|}{ (ii) } \\
\hline Mentorship & 2.473 & 0.002 & $(1.406-4.350)$ \\
\hline Training cost & 0.453 & 0.009 & $(0.250-0.821)$ \\
\hline Acute element & 1.743 & 0.062 & $(0.973-3.121)$ \\
\hline Access to postgraduate training & 0.434 & 0.004 & $(0.248-0.762)$ \\
\hline Academic competitiveness & 1.852 & 0.047 & $(1.008-3.403)$ \\
\hline Year of study & 0.799 & 0.066 & $(0.628-1.015)$ \\
\hline Workload of surgeons & 0.555 & 0.043 & $(0.314-0.982)$ \\
\hline Gender & 0.587 & 0.053 & $(0.342-1.006)$ \\
\hline
\end{tabular}

Bold values indicate stastically significant result $(p<0.05)$ 
Acknowledgments This study was created through the IFMSA Global Surgery Working Group, now InciSioN, the International Student Surgical Network. We thank all the students who participated in the study and who assisted us in publicising the study. We thank Prof. Chris Lavy for input on study design and helping us gain ethical approval, and Dr David Chang for his assistance in data analysis and write-up.

\section{Compliance with ethical standards}

Conflict of interest The authors declare that they have no conflict of interest.

Open Access This article is distributed under the terms of the Creative Commons Attribution 4.0 International License (http://crea tivecommons.org/licenses/by/4.0/), which permits unrestricted use, distribution, and reproduction in any medium, provided you give appropriate credit to the original author(s) and the source, provide a link to the Creative Commons license, and indicate if changes were made.

\section{References}

1. Lillemoe KD, Ahrendt GM, Yeo CJ, Herlong HF, Cameron JL (1994) Surgery-still an" old boys' club"? Surgery 116(2):255-961

2. Barshes N, Vavra A, Miller A, Brunicardi F, Goss J, Sweeney J (2004) General surgery as a career: A contemporary review of factors central to medical student specialty choice. J Am Coll Surg 199(5):792-799

3. Fitzgerald JEF, Tang S-W, Ravindra P, Maxwell-Armstrong CA (2013) Gender-related perceptions of careers in surgery among new medical graduates: results of a cross-sectional study. Am J Surg 206(1):112-119

4. Ravindra P, Fitzgerald JEF (2011) Defining surgical role models and their influence on career choice. World $\mathbf{J}$ Surg 35(4):704-709. https://doi.org/10.1007/s00268-011-0983-0
5. Park J, Minor S, Taylor RA, Vikis E, Poenaru D (2005) Why are women deterred from general surgery training? Am J Surg 190(1):141-146

6. Mayer KL, Perez RV, Ho HS (2001) Factors affecting choice of surgical residency training program. J Surg Res 98(2):71-75

7. Kawase K, Carpelan-Holmström M, Kwong A, Sanfey H (2016) Factors that can promote or impede the advancement of women as leaders in surgery: results from an international survey. World J Surg 40(2):258-266. https://doi.org/10.1007/s00268-015-3332-

8. Healy NA, Cantillon P, Malone C, Kerin MJ (2012) Role models and mentors in surgery. Am J Surg 204(2):256-261

9. Cohen MS et al (2007) Mentorship, learning curves, and balance. Cardiol Young 17(S2):164-174

10. Gargiulo DA, Hyman NH, Hebert JC (2006) Women in surgery. Arch Surg 141(4):405

11. Makama JG, Garba ES, Ameh EA (2012) Under representation of women in surgery in Nigeria: by choice or by design? Oman Med J 27(1):66-69

12. Kaderli R, Guller U, Muff B, Stefenelli U, Businger A (2010) Women in surgery: a survey in Switzerland. Arch Surg 145(11): 1119

13. Holmer H, Shrime MG, Riesel JN, Meara JG, Hagander L (2015) Towards closing the gap of the global surgeon, anaesthesiologist, and obstetrician workforce: thresholds and projections towards 2030. Lancet 385:S40

14. Meara JG et al (2015) Global surgery 2030: evidence and solutions for achieving health, welfare, and economic development. Surgery $158(1): 3-6$

15. Kumar A, Mitra K, Nagarajan S, Poudel B (2014) Factors

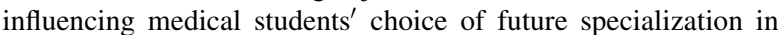
medical sciences: A cross-sectional questionnaire survey from medical schools in china, Malaysia and regions of south Asian association for regional cooperation. N Am J Med Sci 6(3):119

16. Compton MT, Frank E, Elon L, Carrera J (2008) Changes in U.S. medical students' specialty interests over the course of medical school. J Gen Intern Med 23(7):1095-1100

Publisher's Note Springer Nature remains neutral with regard to jurisdictional claims in published maps and institutional affiliations. 